\title{
An Unusual Arrangement of 6 Canals in Maxillary First Molar: A Unique Case Report and Review of Literature
}

\author{
Payal Agarwal ${ }^{1 *}$, Shagun Garg ${ }^{2}$ \\ ${ }^{1}$ Consultant Dental Surgery Department of Oral, Dental \& Craniofacial Care Yashoda Super Specialty Hospital, \\ Kaushambi, Ghaziabad Uttar Pradesh, India \\ ${ }^{2}$ Assistant Professor Department of Conservative Dentistry and Endodontics, D. Y Patil School of Dentistry, Navi \\ Mumbai, Maharashtra, India
}

*Corresponding Author

Payal Agarwal

\author{
Article History \\ Received: 21.11.2020 \\ Accepted: 03.12.2020 \\ Published: 13.12 .2020
}

\begin{abstract}
Root canal therapy, demands adequate accessibility of the pulp chamber followed by a proper biomechanical preparation and three dimensional obturation of the root canals. Permanent maxillary first molar undergoing endodontic management are generally found to have three roots and four canals, although multiple variations in this configuration have been documented in the literature so far. A thorough understanding about predicting and managing such aberrant root canal morphologies is the key to achieve long term success in the field of endodontics. This paper aims to add in the literature, a unique case of permanent maxillary right first molar with a rarest arrangement of 6 root canals. The author(s) have attempted their best to review the literature and enhance the knowledge on aberrant root canal morphology of permanent maxillary molars.
\end{abstract}

Keywords: Permanent Maxillary First Molar, Endodontic, Aberrant, Root Canals.

\section{INTRODUCTION}

A successful root canal therapy (RCT), demands adequate accessibility of the pulp chamber followed by a proper biomechanical preparation and three dimensional obturation of the root canals. In majority, permanent maxillary first molar undergoing RCT are found to have three roots and four canals [1]. Lot of variations have been reported in the literature so far regarding number of roots, number of canals present in each root and incidence of root fusions in permanent maxillary first molars. An understanding about how to predict and manage such anatomical variations in the root canal system is of utmost importance when it comes to the execution and outcome of a RCT [2]. To be more specific although permanent maxillary first molars are known to present with 6 canals, but the present case shows a unique arrangement of 6 canals which is rarely documented in the literature till date.

\section{CASE RePOrT}

A 26 years old female patient reported to our unit with the chief complaint of pain in the upper right back teeth region since last night. She gave the history of mild, aching, on and off pain in the same region while chewing past few days for which she consulted a nearby dentist who did a temporary restoration for the same, 1 week back. Nothing significant was found in her medical history and on intraoral examination her permanent maxillary right first molar was found to have a temporary restoration on the occlusal surface, the tooth was tender on percussion with no mobility present and apparently normal periodontium. It showed lingering positive response to the pulp vitality test. Based on history \& clinical examination a provisional diagnosis of irreversible pulpitis i.r.t. 16 was made. IOPA radiograph (Fig. 1) revealed a radiolucency encroaching the pulp and normal periapex. Correlating the clinical and radiographic observations, the tooth was diagnosed with chronic irreversible pulpitis. Root canal therapy followed by crown was suggested as the treatment of choice and same was opted by the patient. After administering local anaesthesia $(2 \%$ Lidocaine $\mathrm{HCl}$ with 1:80,000 Adrenaline) an access opening was made i.r.t. 16 after deroofing the pulp chamber, orifice

Copyright $\odot 2020$ The Author(s): This is an open-access article distributed under the terms of the Creative Commons Attribution 4.0 International License (CC BY-NC 4.0) which permits unrestricted use, distribution, and reproduction in any medium for noncommercial use provided the original author and source are credited. 
location was done using \#10 K files \& endodontic explorer and 6 root canal orifices were located i.e Mesiobuccal1(MB1), Mesiobuccal-2(MB2), Mesiobuccal-3(MB3), Distobuccal-1(DB1), Distobuccal-2(DB2) \& Palatal(P) (Fig. 3) as described by Vertucci's type I for palatal, type II for distobuccal and additional type XVIII (3-2) for mesiobuccal. Working length was determined with the help apex locator which was re-confirmed using an IOPAR (Fig. 2). Cleaning and shaping was carried out by crown down technique using Rotary protaper gold files up to F1. Root canal irrigation was done with warm 3\% sodium hypochlorite solution using side vented needles followed by saline wash. Each canal was dried with the help of sterile paper points and a dressing of calcium hydroxide was given followed by which the access cavity was sealed with cavit. At the second follow-up, the tooth was found to be asymptomatic, access cavity was opened and the canals were thoroughly irrigated with 17\% EDTA, saline, 3\% sodium hypochlorite \& normal saline. Endodontic sealer was applied over the selected master cones and canals were obturated with corresponding F1 protaper gutta percha points and 6\% \#20 for MB2 using lateral condensation technique (Fig. 4). Post-endo build-up was done with glass ionomer cement and the patient was recalled after 7days for follow-up (Fig. 5). Patient was totally asymptomatic 1 week later, thereby crown preparation was done and the crown was fixed on the subsequent visit.

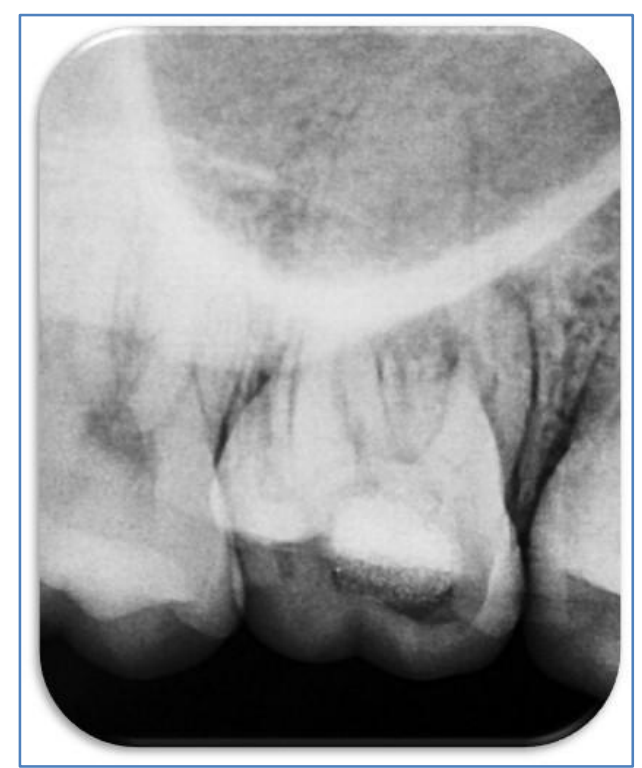

Fig-1: Preoperative IOPA radiograph of maxillary right first molar revealing a radiolucency encroaching pulp

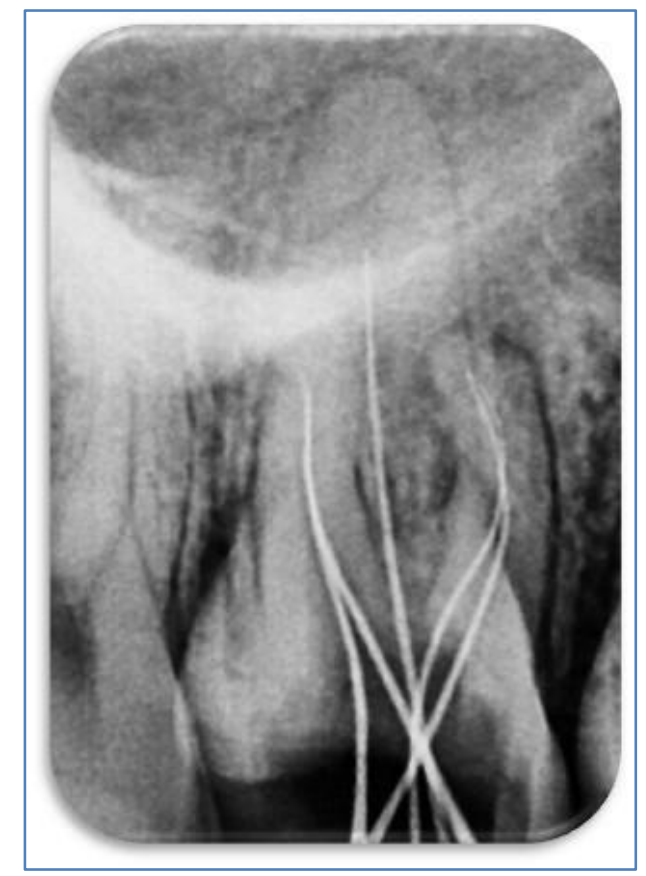

Fig-2: Intraoperative IOPA radiograph depicting the working length 


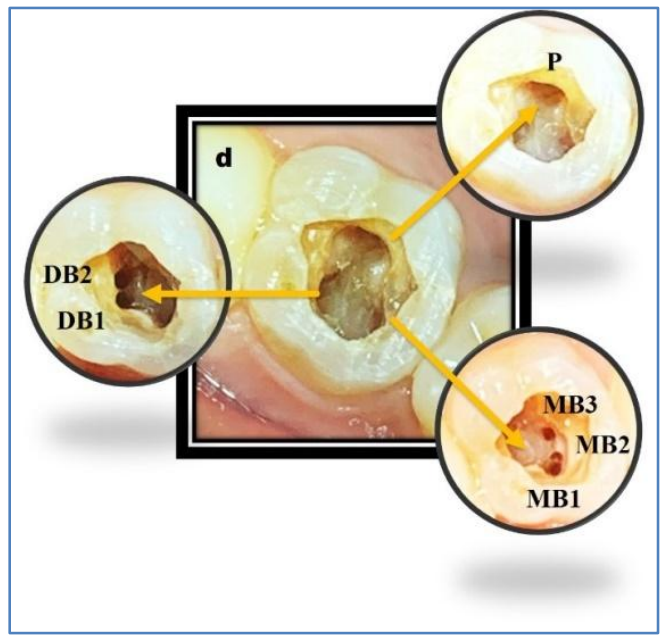

Fig-3: Clinical picture of maxillary right first molar with 6 root canal orifices located (MB1, MB2, MB3, DB1, DB2, P)

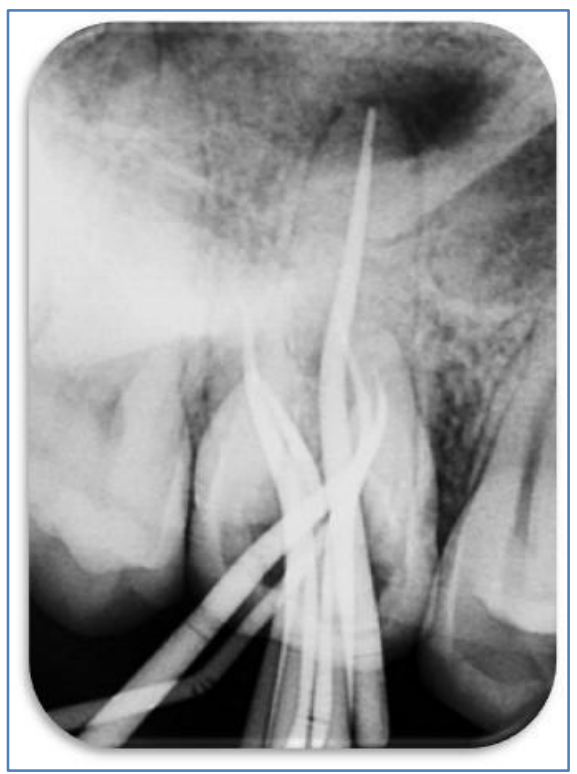

Fig-4: Intraoperative IOPA radiograph with F1 Protaper GP Points and $6 \%$ \#20 for MB2 as master cones

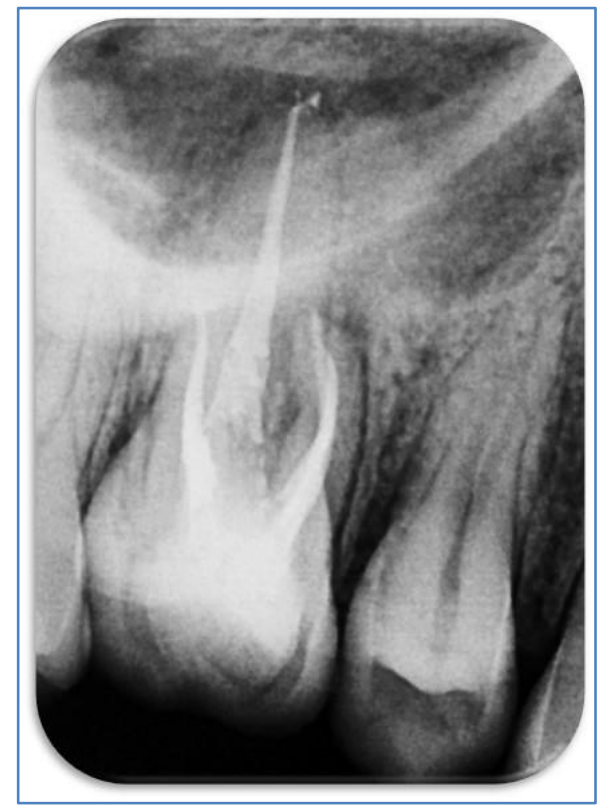

Fig-5: Postoperative IOPA Radiograph in relation to maxillary right first molar 


\section{DISCUSSION}

Inadequate eradication of the microorganisms from the root canals may lead to failure, especially in cases where root canal configuration cannot be assessed preoperatively and aberrant morphology surprise the clinician during the procedure. Therefore it is necessary that a meticulous clinical and radiographic examination of the tooth morphology be performed keeping in mind the possibility of unusual variations which would help in avoiding unintentional treatment failures. Literature depicting cases with aberrant root canal morphology acts as a guide to the clinicians and prepares them for predicting, locating and managing accessory root canals thereby improving the success rate of a root canal treated tooth [3, 4]. Till date, permanent maxillary first molars are already known for their unusual root canal morphologies, especially when it comes to the presence of second mesiobuccal canal, which, according to the literature $[5,6]$ is a very common finding. Majority of such reports have described permanent maxillary first molar with 3 roots and 3 or 4 root canals [7, 8]. Bond et al. [9] reported a permanent maxillary first molar having six root canals i.e. 2 mesiobuccal, 2 distobuccal and 2 palatal canals while in 1983, Martınez-Berna'A et al. [10] also reported three cases of permanent maxillary first molar with six root canals with a rarest configuration i.e. 3 mesiobuccal canals, 2 distobuccal and 1 palatal canal. The present case was found to have similar root canal morphology as reported by Martinez.

A successful RCT demands proper preoperative assessment, identification and location of accessory canal(s). Various methods have been described in the past that can help the clinician in locating of root canal orifices effectively. Multiple pre-operative IOPA radiographs, use of tactile sensation while examining the floor of the pulp chamber with a sharp explorer, troughing with ultrasonic tips, visualizing bleeding points of the canal orifices, staining of the floor of pulp chamber with $1 \%$ methylene blue, performing Champagne bubble test and last but not the least use of magnification with microscopes or surgical loupes are the most commonly known techniques. In the present case, a series of preoperative IOPA radiographs alone revealed the presence of accessory root canals which signifies the importance of radiographic assessment in the detecting of unusual root canal morphology.

It is important for the clinicians to note that the point of care doesn't get over just by locating the accessory canals, every step needs to be performed with strict protocols like instrumentation is always performed with caution to avoid over enlargement of the root canals which may lead to stripping or perforation. In order to prevent shaping aberrations such as ledge formation, root canal transportation, straightening of the root canal, loss of working length and instrument separation [11], initial exploration of the canals is to done with smaller files of size 10 -15 or lesser followed by creation of a glide path using flexible nickel titanium hand or rotary files of suitable taper with adequate lubrication. Hourglass shape of the mesial root should be kept in mind which makes it vulnerable for perforation at the mid-section. Thus the root canal cleaning and shaping should be done conservatively and with utmost caution to achieve long term success.

\section{Conclusion}

It is evident from the present case report that the clinicians, while keeping in mind the general considerations about the root canal morphology, also should be aware and knowledgeable predict the incidence of accessory root canals at the same time. Preoperative radiographic assessment of the tooth of concern and its integration with a careful clinical examination should be considered as a tool of utmost importance in terms of successful endodontic therapy. As discussed in this article, literature is sparse regarding endodontic management of an unusual root canal configuration of a maxillary first molar with three roots and six canals (MB1, MB2, MB3, DB1, DB2, P). Further studies are required to report more of such rare cases in the literature and help the clinicians to achieve more predictable endodontic results.

\section{REFERENCES}

1. Walton, R. (2009). Torabinejad M. Principles and practice of endodontics. Saunders, Philadelphia, 30, 306.

2. Kulid, J. C., \& Peters, D. D. (1990). Incidence and configuration of canal systems in the mesiobuccal root of maxillary first and second molars. Journal of endodontics, 16(7), 311-317.

3. Vertucci, F. J. (1984). Root canal anatomy of the human permanent teeth. Oral Surgery, Oral Medicine, Oral Pathology and Oral Radiology, 58(5), 589-599.

4. Pecora, J. D., Woelfel, J. B., Sousa Neto, M. D., \& Issa, E. P. (1992). Morphologic study of the maxillary molars. Part II: Internal anatomy. Brazalian Dental Journal, 3(1), 53-7.

5. Çalişkan, M. K., Pehlivan, Y., Sepetçioğlu, F., Türkün, M., \& Tuncer, S. Ş. (1995). Root canal morphology of human permanent teeth in a Turkish population. Journal of endodontics, 21(4), 200-204.

6. Buhrley, L. J., Barrows, M. J., BeGole, E. A., \& Wenckus, C. S. (2002). Effect of magnification on locating the MB2 canal in maxillary molars. Journal of endodontics, 28(4), 324-327.

7. Chande, K. P., Manwar, N. U., Chandak, M. G., \& Lokade. (2013). J. Maxillary and mandibular first molar with five root canals diagnosed with cone-beam computed tomography: A review and two case reports. Journal of Dental and Medical Sciences 10(5), 6-11. 
8. Çalişkan, M. K., Pehlivan, Y., Sepetçioğlu, F., Türkün, M., \& Tuncer, S. Ş. (1995). Root canal morphology of human permanent teeth in a Turkish population. Journal of endodontics, 21(4), 200-204.

9. Bond, J. L., Hartwell, G., \& Portell, F. R. (1988). Maxillary first molar with six canals. Journal of endodontics, 14(5), 258-260.

10. Martínez-Berná, A., \& Ruiz-Badanelli, P. (1983). Maxillary first molars with six canals. Journal of endodontics, 9(9), 375-381.

11. Calberson, F. L., De Moor, R. J., \& Deroose, C. A. (2007). The radix entomolaris and paramolaris: clinical approach in endodontics. Journal of endodontics, 33(1), 58-63. 